\title{
Estrategia metodológica para desarrollar la competencia del manejo de la información en estudiantes universitarios
}

\section{Methodological strategy to develop the competence of information management in university students}

\author{
Verónica Rodríguez Arboleda \\ Universidad Internacional SEK Ecuador, Ecuador \\ Yamirlis Gallar Pérez \\ Universidad Internacional SEK Ecuador, Ecuador \\ Enrique Aurelio Barrios Queipo \\ Universidad Internacional SEK Ecuador, Ecuador
}

Autor para correspondencia: veronica.rodriguez@uisek.edu.ec

Fecha de recepción: 10 de Septiembre de 2016 - Fecha de aceptación: 01 de Noviembre de 2016

\section{Resumen}

El proceso de enseñanza aprendizaje tiene una estructura y un funcionamiento sistémico, la calidad de su componente de ejecución depende del procesamiento de la información, ya que su uso adecuado posibilita la participación activa de los estudiantes en la producción de resultados de aprendizaje. El presente estudio muestra una estrategia metodológica para el manejo de la información que concientiza sobre necesidad de acceder y utilizar diversas fuentes, respetando la propiedad intelectual de sus autores. Se utiliza la Investigación Acción Participativa y se implementa un conjunto de acciones de manera que las prácticas investigativas se reviertan en la apropiación de destrezas para adquirir, analizar, evaluar, organizar, procesar y comunicar mediante el uso de tecnología.

Palabras claves: manejo de información; competencia; estrategia metodológica

\begin{abstract}
The teaching-learning process has a structure and systemic functioning, the quality of its execution component depends on the information processing, its proper use enables the active participation of students in the production of learning outcomes. This study presents a methodological strategy for information management that awareness about the need to access and use a variety of sources, respecting the intellectual property of their authors. Participatory Action Research is used to implement actions so that research practices are reversed in the appropriation of skills to acquire, analyze, evaluate, organize, process and communicate using technology.
\end{abstract}

Key words: information management; competition; methodological strategy 


\section{Introducción}

Los estudiantes de esta generación nacieron con la tecnología, ésta es parte de sus vidas, la utilizan para la educación, diversión y socialización con sus pares. El Internet se ha convertido en su principal fuente de consulta, a la que acuden para realizar sus tareas, que en muchos casos se ha reducido a una actividad de copiar y pegar la primera información que encuentran, lo que evidencia que los estudiantes no tienten el conocimiento y las destrezas para manejar eficientemente la información. Se presenta entonces la necesidad eminente, que en el proceso de enseñanza-aprendizaje los docentes guiemos a los estudiantes en el manejo adecuado de la información, lo que se puede lograr a través de la aplicación de una estrategia metodológica adecuada que tome en cuenta el contexto en que se desarrollan los estudiantes, los recursos con los que cuentan y sus intereses de aprendizaje. Bajo estas consideraciones, los autores hemos elaborado una estrategia metodológica, para apoyar a los estudiantes de sistemas de la Universidad SEK del Ecuador, en el desarrollo de sus capacidades y habilidades para manejar la información.

El conjunto de conocimientos, habilidades y actitudes que el estudiante aplica para identificar lo que quiere investigar, buscar efectivamente la información, determinar si esa información es pertinente y finalmente convertirla en conocimiento útil para solucionar problemas de Información de la vida real es lo que se conoce como competencia para el manejo de la Información o CMI.

La estrategia que se presenta ha sido diseñada para desarrollar esta competencia, y consiste en una secuencia de pasos que el estudiante sigue desde que tiene la necesidad de consultar hasta que finaliza su investigación documental y obtiene un producto final que lo socializa.

\section{Desarrollo}

La información es un bien preciado en la Sociedad de la Información y el Conocimiento, y su importancia ha sido reconocida a todo nivel y área de acción tanto mundial como regionalmente, por ello se ha considerado importante para los autores, partir el presente artículo mencionando y analizando los acuerdos o recomendaciones de organismos internacionales, y las políticas y planes del estado ecuatoriano que hacen referencia a su uso en la educación.

La Organización de las Naciones Unidas para la Educación y Diversificación, la Ciencia y la Cultura (UNESCO), en una de sus recomendaciones sobre la promoción y el uso del plurilingüismo y el acceso universal al ciberespacio en el desarrollo de los contenidos de dominio público dice:

"Los Estados Miembros y las organizaciones internacionales deberían promover y facilitar la "alfabetización electrónica", lo que incluye actividades encaminadas a divulgar las tecnologías de la información y la comunicación e infundir seguridad y confianza en su aplicación y utilización. El desarrollo del "capital humano" de la sociedad de la información, y en especial una enseñanza abierta, integrada e intercultural combinada con la adquisición de las aptitudes necesarias para manejar las tecnologías de la información y la comunicación reviste 
una importancia capital. La formación en esa materia no debería circunscribirse a la adquisición de competencias técnicas, sino que debería dar también cabida a una sensibilización a principios y valores éticos." (Organización de las Naciones Unidas para la Educación y Diversificación, 2003).

Esta recomendación es totalmente aplicable en los procesos de enseñanza aprendizaje, en el que docente no instruye sino forma al estudiante y se preocupa no solo de llegar con el conocimiento, sino en desarrollar habilidades y valores que le permitan manejar la información de forma eficiente utilizando la tecnología en un marco de respeto a la propiedad intelectual.

Analizando el ámbito del país, en el Ecuador es un objetivo nacional promover espacios de educación para el intercambio de conocimientos, como lo indica en el literal a) del objetivo 4.3 del Plan Nacional del Buen vivir, que dice, es objetivo nacional:

"Democratizar el acceso al conocimiento, fortaleciendo los acervos de datos, la información científica y los saberes diversos en todos sus formatos, desde espacios físicos y virtuales de libre acceso, reproducción y circulación en red, que favorezcan el aprendizaje y el intercambio de conocimientos". (Secretaría Nacional de Planificación y Desarrollo, 2013).

De lo que se puede destacar, que el estado promueve que todos los ecuatorianos tengamos acceso a la información desde distintos medios como el internet, con el fin de favorecer el aprendizaje. Es esencial entonces, que desde el proceso de enseñanza aprendizaje haya un interés bien marcado desde las materias que se reciben en cuáles son los requisitos que deben apropiarse los estudiantes para el manejo de la información para aprender y generar conocimientos, y sería apropiado que la Universidad sea baluarte de esta aspiración.

Si se considera el contexto de la Educación Superior del Ecuador y el Reglamento de Régimen Académico que lo rige, en el artículo 16, literal 3) al describir los componentes de las actividades de aprendizaje, se define que:

"Componente de aprendizaje autónomo. - comprende el trabajo realizado por el estudiante, orientado al desarrollo de capacidades para el aprendizaje independiente e individual del estudiante. Son actividades de aprendizaje autónomo, entre otras: la lectura; el análisis y comprensión de materiales bibliográficos y documentales, tanto analógicos como digitales; la generación de datos y búsqueda de información; la elaboración individual de ensayos, trabajos y exposiciones." (Consejo de Educación Superior, 2013).

Esta aspiración desde los documentos rectores, implica que el estudiante universitario debe saber manejar de forma eficiente la información para que tenga éxito en su aprendizaje, entonces es responsabilidad de los docentes que desde las aulas ayudemos a nuestros estudiantes a desarrollar habilidades y conocimientos para manejar la información de tal manera que su aprendizaje sea significativo.

Esta aspiración se manifiesta además desde el Modelo Educativo de la Universidad Internacional SEK del Ecuador, donde se precisa entre sus objetivos que: "Promover la mejora sostenida de la calidad educativa que oferta la UISEK, mediante un enfoque visionario y una 
formación basada en competencias, que responda a las necesidades de desarrollo del país, la sociedad y la institución "(UISEK, 2015):

El modelo, recoge también como referencia epistemológica, lo que la Comisión Europea en el 2004 definió como competencia: "Se refiere a una combinación de destrezas, conocimientos, aptitudes y actitudes, y a la inclusión de la disposición para aprender, además del saber cómo. (SEK, 2011)

Y, en su Reglamento de Régimen Académico, en el Título II que hace referencia a Misión, ideario y filosofía educativa de la UISEK en su artículo 4 dice:

"La Institución Internacional SEK, comunidad humana, está abierta a toda experiencia educativa encaminada al logro de sus fines." (UISEK, 2015)

En el artículo 5, dice (UISEK, 2015): "La metodología utilizada en las clases es creativa y participativa, de manera flexible y dinámica, incluyente, eficaz y eficiente, evitando el concepto de confrontación profesor alumno y fomentando el de cooperación profesor-alumno. Esta mística, compartida por todo el claustro académico, facilita el que la actividad docente se convierta en un proceso globalizado y comprensible para los alumnos."

Estas concepciones epistemológicas provocan una interpretación al proceso enseñanzaaprendizaje en el Área de Sistemas Informáticos, lo que estimulan a elevar la calidad académica desde esta área y que por su naturaleza adquiere un carácter interdisciplinario para el resto. Se pueden aplicar con libertad estrategias metodológicas para desarrollar sus competencias y mejorar el desempeño de los estudiantes, por lo tanto, se podrá aplicar la estrategia diseñada.

\section{Fundamentos teóricos sobre la competencia para el Manejo de la Información}

Según Benito Morales (2000) en su artículo "Estrategias y modelos para enseñar a usar la información" indica que, para los psicólogos y documentalistas, "lo importante no es memorizar/almacenar la información, sino desarrollar habilidades/técnicas de recuperación, comprensión y transferencia de la información", con lo que concordamos plenamente desde nuestro punto de vista como profesionales de sistemas y de educación.

Según Eduteka (2006), la Competencia para Manejar Información (CMI) se define como el conjunto de conocimientos, habilidades y actitudes que el estudiante debe poner en práctica para identificar lo que necesita saber en un momento dado, buscar efectivamente la información que esto requiere, determinar si esa información es pertinente para responder a sus necesidades y finalmente convertirla en conocimiento útil para solucionar problemas de información en contextos variados y reales de la vida cotidiana.

A partir del análisis de este concepto se puede decir que los estudiantes alcanzaron la competencia para manejo de la información (CMI), cuando hayan adquirido los conocimientos y desarrollado las habilidades que deben poner en práctica para afrontar sus necesidades de información (EISENBERG, M.,sf)g; y, además, exhiban una serie de actitudes que demuestren que, siempre que deban hacerlo, están dispuestos a utilizar sus conocimientos y a realizar de la 
mejor manera las tareas requeridas. Hasta tanto ellos no demuestren estas actitudes, no se puede decir que son competentes, aunque tengan las capacidades para atender sus necesidades de información. (Edukeka, 2006)

Se puede indicar que:

Competencia $=$ Capacidades + Actitudes

Capacidades $=$ Conocimientos + Habilidades

Entonces:

Competencia $=$ Conocimientos + Habilidades + Actitudes

\section{Modelos para el Desarrollo de la Competencia en el Manejo de la Información.}

Del análisis de información referente al tema, se han encontrado modelos diseñados para el desarrollo del CMI a ser implementados en el proceso de enseñanza aprendizaje en norteamericana y europea.

Tabla 1: Modelos para el desarrollo de CMI

GAVILÁN
$\begin{aligned} & \text { Desarrollo de Competencia para el Manejo de la Información } \\ & \text { (Colombia) }\end{aligned}$




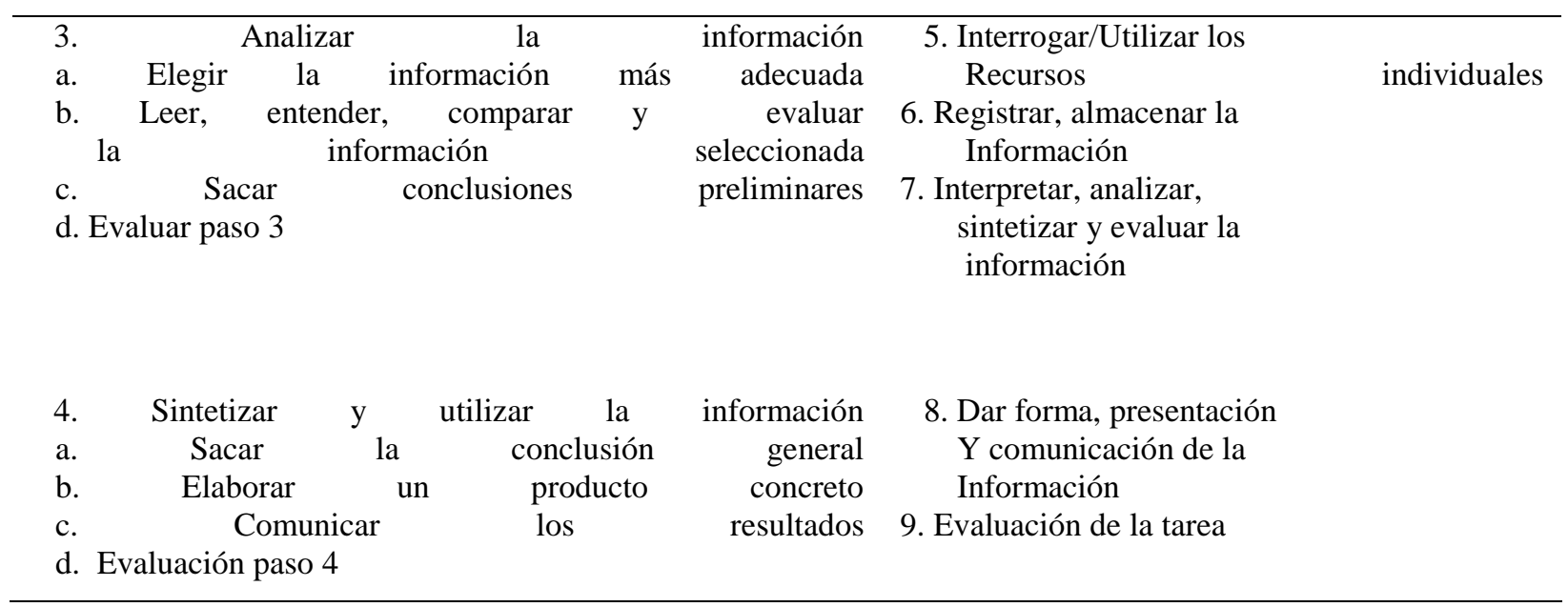

Fuente: Eduteka.

Estos modelos han sido creados en base a los métodos de enseñanza y costumbres de esos países y nos parecen muy interesantes, además de ser un punto de partida para nuestra investigación, no obstante, están ajenos a nuestro país y contexto universitario, por lo cual se desarrolla una metodología que se ajuste a los recursos y formación de los estudiantes de la UISEK.

\section{Competencia para el Manejo de la información de los estudiantes de la UISEK.}

El Internet se ha convertido en la primera fuente de información de los estudiantes, por la facilidad de uso y acceso rápido a gran variedad de información, pero éstas características, han generado el facilismo en los alumnos; para la gran mayoría de los cuales, consultar es copiar la primera información que despliega el buscador y pegarla como producto final de su consulta, sin realizar un verdadero proceso de manejo de la información, que parta de una búsqueda eficiente de información y propicie la generación de conocimiento.

Por parte de los docentes se ha podido evidenciar que en su mayoría los estudiantes solo reproducen la información, no la procesan, menos aún aportan con un verdadero análisis, síntesis y reflexión. De la Parra Jara, N. (2005).

Esta problemática motivó a los autores a diseñar una estrategia que ayude a los estudiantes a manejar de forma eficiente la información.

\section{Diseño de una estrategia metodológica para el desarrollo de la competencia del manejo de la información}

Alineadas a los objetivos institucionales, basadas en situación problémica diagnosticada y consientes de la responsabilidad que como docentes tenemos de formar estudiantes competentes para la vida y en los contextos en que se desenvuelven, hemos diseñado una estrategia metodológica para el desarrollo de la competencia de manejo de la información, tomando como referencia modelos desarrollados en Norteamérica y Europa. 
La estrategia metodológica desarrollada sugiere una secuencia de pasos para manejar de forma eficiente la información lo que permitirá desarrollar el CMI de los estudiantes.

1. Definir el tema a investigar. Ya sea como fruto de auto motivación por conocer sobre un tema en particular o por una necesidad de responder a una tarea encomendada, se deberá definir el tema a investigar de forma clara, concreta y delimitada, identificando la necesidad a la que responderá la investigación y el contexto en el que se desarrollará, estos dos criterios permitirán también determinar los subtemas a consultar.

2. Buscar la información. La información existe en múltiples fuentes o recursos y en cantidades exorbitantes que ha sido almacenada o publicada en el transcurso del tiempo por diferentes autores y en diversos idiomas o formatos, por lo cual para realizar una búsqueda eficiente se deberá considerar:

Estrategias de búsqueda que permitan encontrar información de calidad que se ajuste a los requerimientos.

Para búsquedas en internet se sugiere:

$\checkmark$ Determinar fuentes de consulta. - libros, paperas, páginas web, artículos, blogs, conferencias, congresos, etc.

$\checkmark$ Definir recursos para consultar. - se deberá saber con qué herramientas se puede buscar, por ejemplo, utilizar: Google, Google escolar o Académico, para búsquedas en bibliotecas, libros, bases de datos digitales, papers.

$\checkmark$ Decidir en qué lenguaje se desea buscar la información, lo cual delimitará el ámbito de búsqueda y por tanto se tendrá los resultados esperados.

$\checkmark$ Definir período de publicación. - Es importante buscar información que haya sido publicada recientemente, delimitar la fecha permitirá tener como resultados de la búsqueda, información con validez oportuna.

$\checkmark$ Utilizar cadenas de búsqueda. - para no divagar con la búsqueda y obtener resultados lejanos a lo que se requiere, se deberá utilizar cadenas de búsqueda que filtran la información a encontrar, las siguientes son cadenas válidas:

Tabla 2: Cadenas de búsqueda en Internet

\begin{tabular}{lll}
\hline Objetivo & Búsqueda & Cadena \\
\hline Buscar palabras exactas & Ingrese las palabras entre comillas & "frase“ \\
Buscar una y otra palabra & Ingresa AND entre las palabras & palabra AND palabra \\
Buscar una u otra palabra & Ingresa OR entre las palabras & palabra OR palabra \\
Excluir una o unas palabras & Digitar un signo menos delante de la o las palabras & -palabra \\
\hline
\end{tabular}

Realizado por: Verónica Rodríguez

Como explicación de los resultados obtenidos de la aplicación de la estrategia de búsqueda, se recomienda indicar los criterios de inclusión y exclusión que se eligieron para seleccionar la información, por ejemplo: indicar solo el tipo de fuente elegida, el período, el idioma, o que información se descarta que no aporte a la investigación en el contexto que se remarcó. EISENBERG, M., 
3. Analizar y sintetizar la información.

La información obtenida en la búsqueda, será abundante, y para sintetizarla será necesario seguir estos pasos:

- Organizar la información. - agrupar la información según la temática o alguna característica afín y determinar claramente la fuente de cual se obtuvo y sus autores para referenciarlos con total transparencia y no caer en el plagio.

- Comparar y evaluar la información. - identificar la información que más se ajuste a los requerimientos de la búsqueda y de igual forma determinar la que no sea representativa.

- Extraer la información relevante. - De la información seleccionada se deberá extraer la que verdaderamente aporte de forma sustancial a la investigación, y de ella describir algunos criterios sus fuentes tales como:

Fecha de extracción

$\checkmark \quad$ Fuente de la publicación elegida

$\checkmark \quad$ Título de la publicación

$\checkmark \quad$ Origen, autor o autores de la publicación

$\checkmark \quad$ Año de la publicación

- Sintetizar la información. - realizar un resumen de toda la información encontrada incluyendo por su puesto el aporte personal.

4. Utilizar la información. Sea cual haya ha sido la razón que motivó la investigación y después de que se ha realizado un trabajo minucioso de búsqueda, selección, análisis y síntesis de la información, amerita:

- Elaborar un producto final. - que refleje el trabajo de investigación realizado y el aporte personal y este resultado constará claramente las referencias de las fuentes consultadas.

- Presentar o socializar el producto. - El trabajo realizado, tendrá más relevancia si es un aporte para el conocimiento de otras personas, para lo cual es importante que sea compartido o socializado de alguna manera o en cualquier medio o recurso.

Con la aplicación de esta estrategia metodológica se estará implícitamente desarrollando en el estudiante la Competencia para el Manejo de la Información (CMI).

\section{Resumen de la Estrategia metodológica para el desarrollo de CMI.}

A continuación, se esquematiza la estrategia propuesta: 
1. Definir el tema a investigar

a. Identificar necesidad y contexto.

b. Determinar subtemas relacionados

2. Buscar la información

a. Determinar estrategias de búsqueda

i. Definir fuentes de consulta

ii. Recursos consultados

iii. Lenguaje

iv. Período de publicación

v. Cadenas de Búsqueda

b. Selección de la Información

i. Determinar criterios de inclusión y exclusión

3. Analizar y sintetizar la información
a. Organizar la información
b. Comparar y evaluar la información
c. Extraer la información relevante
d. Elegir la información más adecuada
e. Sintetizar la información

4. Utilizar la información
a. Elaborar un producto final
b. Presentar o socializar el producto

Como resultado de la Metodología aplicada el estudiante deberá adjuntar al producto final de la investigación la siguiente ficha que registra y evidencia los pasos seguidos en la investigación:

Tabla 3: Ficha de Aplicación de la Estrategia Metodológica para desarrollo CMI

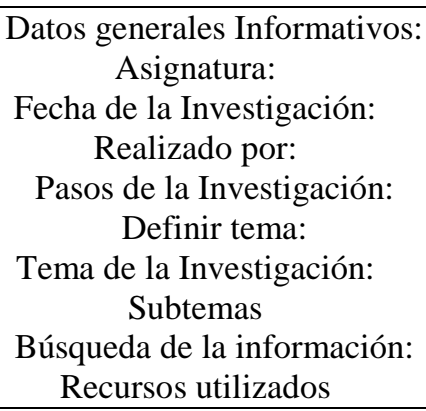




Fuentes de Consulta
Lenguajes en los que se buscó
Períodos de publicación en los que se buscó
Criterios de exclusión
Cadenas de búsqueda
Sintetizar y organizar
Autor o autores de la publicación y origen, Año de la publicación, Título de la publicación, fuente de la
publicación, Fecha de extracción
Resumen
Utilización de la información (indicar cómo se publicará o socializará)

Realizado por: Verónica Rodríguez

Esta estrategia cumple su objetivo ya que conduce al estudiante de una forma sistemática a manejar la información como se argumenta a continuación:

- En principio concientiza al estudiante sobre la importancia de saber buscar la información y de utilizar recursos para que la búsqueda sea más eficiente, utilizando diferentes buscadores y sus opciones avanzadas como filtros para fechas, idiomas, etc.

- Esta estrategia canaliza los esfuerzos del estudiante para buscar información veraz, actualizada y relevante para sus trabajos al hacerlo por ejemplo en artículos científicos, paperas, libros.

- Conforme se aplica la metodología los estudiantes toman destreza para manejar la información, comparando entre varias fuentes y seleccionando ideas relevantes.

- Desarrollará el respeto a la propiedad intelectual ya que deberán referenciar a sus autores.

- La metodología genera que los estudiantes sinteticen la información, reflexionen y realicen aportes personales al elaborar resúmenes de sus trabajos.

- La metodología guía para que los resultados de sus investigaciones socialicen con sus compañeros de diferentes maneras.

En el análisis de la estrategia se puede considerar algunas desventajas que se pueden presentar al momento de aplicar la estrategia, como son:

- En un principio va a chocar con los hábitos que tienen los estudiantes al momento de consultar, lo que provocará que les tome tiempo en poner en práctica cada uno de los pasos y por lo tanto obtener el resultado de la consulta

- Demanda mayor esfuerzo de los estudiantes al buscar en más fuentes y evaluar que éstas sean confiables y que tengan autores que se puedan referenciar, lo que puede desanimar al estudiante si no valora el resultado obtenido.

- La estrategia exigirá mayor trabajo al docente, que debe realizar un seguimiento constante para poder evaluar objetivamente el resultado obtenido en cada paso. 


\section{Conclusiones}

Desde las concepciones teóricas abordadas, se puede concluir que la competencia para manejo de la información es consecuente con el desarrollo de las habilidades para adquirir y evaluar, organizar y mantener, interpretar, procesar y comunicar, la información, utilizando la tecnología.

La estrategia metodológica desarrollada es producto de combinar métodos de otros autores estudiosos del tema del CMI, la motivación de los docentes por ayudar a los estudiantes a que manejen de forma consiente y eficiente la información, así como la experiencia en el contexto en el que aplicará.

La estrategia metodológica aplicada progresivamente podrá romper paradigmas en los estudiantes y generar hábitos que mejoren el proceso de enseñanza aprendizaje y permitirá desarrollar su competencia para el manejo de la información, para realizar sus tareas de investigación de forma más eficiente y de calidad.

\section{Bibliografía}

AASL, (2002). Los nueve estándares de la Competencia en Manejo de Información (CMI)

Benito Morles, F. G. (2000). Estrategias y modelos para enseñar a usar la información.

Consejo de Educación Superior. (2013). Reglamento de Régimen Académico.

De la Parra Jara, N. (2005). El desarrollo de destrezas integrales para lograr la competencia en el manejo de la información. REXE: Revista de estudios y experiencias en educación, 4(7), 141-150.

Eduteka. (2006). Tecnologías de la información y las comunicaciones para la enseñanza básica y media: La enseñanza de la competencia en el manejo de la información mediante el modelo BIG 6.

EISENBERG, M., \& Berkowitz, B. El modelo Big Six para la solución de problemas en información. Portal Eduteka: Tecnologías de Información y Comunicaciones para la Enseñanza Básica y Media, no, Disponible en: http://www. eduteka. org/tema_mes. php3.

Organización de las Naciones Unidas para la Educación y Diversificación, 1. C. (2003). Recomendación sobre la promocion y el uso del plurilingüismo y el acceso universal al ciberespacio.

Muelas, E. Gestión de la Información: organización, búsqueda y recuperación en Internet.

Secretaría Nacional de Planficación y Desarrollo. (2013). Plan Nacional del Buen Vivir.

SEK, U. I. (2011). Modelo Educativo basado en Competencias de Formación Integral. 
UISEK, U. I. (21 de enero de 2015). Reglamento Académico. Quito. 